\title{
Multicenter bridge to transplantation with the HeartMate assist device: Evaluation from another perspective
}

Jack G. Copeland, MD

See related articles on pages 227 and 440 .
From the Department of Cardiothoracic Surgery, The University of Arizona Health Science Center, Tucson, Ariz.

Received for publication July 5, 2001; accepted for publication Aug 15, 2002.

Address for reprints: Jack G. Copeland, MD, Department of Cardiothoracic Surgery, The University of Arizona Health Science Center, PO Box 245071, Tucson, AZ 85724 (E-mail: jgc@u.arizona.edu).

J Thorac Cardiovasc Surg 2003;125:228-30

Copyright () 2003 by The American Association for Thoracic Surgery

0022-5223/2003 $\$ 30.00+0$

doi: $10.1067 / \mathrm{mtc} .2003 .68$

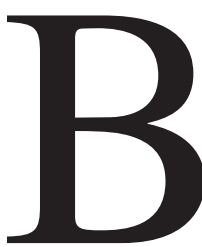

ridge to transplantation has been a scenario for the use of a variety of mechanical support devices since the mid-1980s. Nearly every device studied led to survival to transplantation in the range of $60 \%$ to $70 \%$ and survival to discharge after transplantation of $50 \%$ to $60 \%$ of the total population. Selection has not been standardized, but in general the patient population, in the absence of device support, has been at very high risk. Several thousand lives have been saved, and much experience has been gained in the use of artificial hearts, left ventricular assist devices (LVADs), and biventricular assist devices (BiVADs). This experience will lead to future use of mechanical support devices as permanent definitive therapy for a population of patients with heart failure in the United States that has most often been estimated to be in the range of 30,000 to 60,000 patients per year. Definitive use of device support is underway in small numbers of patients and appears very promising. Bridge to transplant studies such as the report by Frazier and associates ${ }^{1}$ in this Journal are therefore of vital concern to the thoracic surgery community.

The report by Frazier and colleagues ${ }^{1}$ of 280 implants of the HeartMate vented electric LVAD (Thermo Cardiosystems, Inc, Woburn, Mass) at 24 centers in the United States represents a major effort by a group of excellent surgeons. It documents that, of the total number of patients, $67 \%$ survived to transplantation and $56 \%$ (84\% of those undergoing transplantation) lived for at least 1 year after transplantation compared with $33 \%$ survival to transplant and $21 \%$ (63\% of those transplanted) survival for 1 year after transplantation in a group of 48 matched historical controls. "Outpatient enrollment" for implanted patients was 70\% (160 patients) and "full outpatient status" was attained by $41 \%$ (115 patients), demonstrating that out-of-hospital life was possible in a large proportion of the study group. For these reasons, it must be regarded as an important study supporting the concept of bridge to transplantation and the promise of using mechanical support devices as alternatives to transplantation. But, like many previous sponsored mechanical support device studies, it suffers from imperfections that result from study design and incomplete information and thus at once tantalizes and frustrates the critical reader. To encourage such readers to be more accepting of the truths of this article and aware of the shortcomings, I propose a review of some of the limitations of this and other "artificial heart" studies.

First there is the problem of "a controlled study." The current "evidence based" practice of medicine and surgery is founded on the randomized prospective trial. The Food and Drug Administration (FDA) has reluctantly accepted historical controls, such as those used in this study, in bridge to transplant studies. The ethical problem in mechanical support device research has been that the surgeon, who is trying to maximize benefit and minimize risk in his critically ill patient, cannot ask such a patient to be randomized to the non-device group. This is especially true the sicker the patient becomes and the more positive the surgeon is of the benefit of his device and ultimate goal (bridge to transplantation). It is less true in more stable patients with less well-defined goals. For instance, in this study, with patients receiving the implant having a mean cardiac index of $1.67 \mathrm{~L} \times \min ^{-1} \times \mathrm{m}^{-2}$, a systolic blood pressure of $75 \mathrm{~mm} \mathrm{Hg}$, a wedge pressure of $27 \mathrm{~mm} \mathrm{Hg}$, and all patients having maximal inotropic support with $49 \%$ on intra-aortic balloon pumping, the principal investigators would effectively be asking $50 \%$ of their patients to 
make the "ultimate sacrifice" for the sake of a controlled study while the other $50 \%$ would have a reasonable chance of going on to heart transplantation. In contrast, the randomized REMATCH trial is enrolling functional class IV patients who are more stable. ${ }^{2}$ In this group of patients who are not transplant candidates, randomization is ethically reasonable since the outcome for continued medical therapy versus definitive device therapy is unknown.

It is not surprising to the clinician that there was a huge difference in survival in this report between the treated patients and the historical controls because he or she is experienced enough to know that the mortality in these very sick patients is very high. Two other previously published multicenter bridge to transplant studies of devices (Novacor $^{3}$ [Baxter Healthcare Corp, Novacor Div, Oakland, Calif] and CardioWest ${ }^{4}$ [CardioWest Technologies, Inc, Tucson, Ariz]) used historical controls. They, too, found highly significant survival differences favoring the patients receiving the device. Although the science of these studies is imperfect, the benefit of these devices in prolonging life is as certain as we can make it.

Definition and interpretation of "adverse events" is another stumbling block in artificial heart papers. Frazier's report states that patients "had an acceptably low incidence of adverse events." It goes on to say, “. . . our assignment of adverse events to the device or to the patient in this study was subjective...," but they did report the total incidence of events according to the definitions set by the study.

If we look at bleeding as an example, the problems of definition and interpretation become evident. In this study, the adverse event "bleeding" is defined as requiring a reexploration or causing death. Volumes and rates of blood loss are not quantitated. Thus, lesser amounts of blood loss, which may have been significant, are ignored. Of 280 patients, 133 (48\%) met the adverse event definition of bleeding. Eighty-three percent of the "bleeding" was during the first 5 postimplant days. Device-related bleeding (coming from the device or the pocket) was reported in 31 patients $(11 \%)$. What does this say? As the authors noted, "the incidence of bleeding was relatively high." Given the study definition that ignores rate of bleeding or total volume of bleeding, it must be concluded that bleeding is a significant problem in this study. One can only guess what a more inclusive definition might have yielded. Sixty percent would be a conservative guess.

Was all of this bleeding solely because of the severity of patient disease, or could it be related to the device itself? Device-related bleeding is rarely mentioned in other reports, yet it accounts for nearly one fourth of the bleeding in this study. Whether one agrees with the reporting definitions or the subjective division between device related and not device related that is mandated by the FDA, it is clear in this report that bleeding is a problem and that it may be related to the device. The Columbia group found evidence of a "low grade DIC" (increased thrombin generation and fibrinolysis) in HeartMate patients compared with heart failure patients and concluded that there was therefore a potential for increased bleeding. ${ }^{5}$ This then leads to speculation that if the "biologic neoinitma" of the HeartMate device confers a status that discourages thromboembolism, that is, creates a "hypocoagulable" state at the time of implantation, would this explain the increased bleeding? We do not know the answer to this complex question and have little information to interpret other questions that arise.

Was this bleeding excessive? The Novacor FDA study, ${ }^{6}$ using a broader definition of bleeding, reported a $39.7 \%$ total incidence and, also using a broader definition, the CardioWest national study found a 33\% total incidence of bleeding. ${ }^{4}$ Both of these are considerably lower than the narrowly defined $48 \%$ in this study. Notwithstanding these numbers, an independent retrospective comparison of significant numbers of Thoratec, Novacor, and HeartMate implantations failed to show any significant difference among those 3 devices in perioperative bleeding. Unfortunately, bleeding was not formally defined in that study. ${ }^{7}$ No other comparative study has been done.

Our analysis of the bleeding adverse event in this report leads to a general impression and a number of unanswered questions that were not part of the original investigational device exemption (IDE) study. So it is with many of the other adverse events in this and other IDE reports. The studies were designed to define efficacy and safety long before the data were available to provoke a host of new questions. To further look into the bleeding question will require additional studies.

Other adverse events that seem important in this report include the high rate of driveline infections (90 patients, $32 \%$ ). A report from Cleveland found $28 \%$ of HeartMate patients had driveline infections. ${ }^{8}$ The issue of the effect of this device with "biologic lining" on the inflammatory and immune systems and consideration of other factors that might cause driveline infections were not directly addressed. One is again left with a nagging question.

Finally, the large number ( 435 events or 1.2 per 100 days of support) of device malfunctions is remarkable. Certainly prospective users and even participants in the study should be aware that this is an issue even though most events were related to external components and there were no serious complications in this study.

It is expected, in a company-sponsored study regulated by an IDE from the FDA and conducted by investigators who believe in the merits of the technology, that bias of various origins may influence the data. This is true for all company-sponsored FDA-regulated device studies. It is also impossible for the investigators to deal with unanticipated questions that arise during the course of the study. Proving 
safety and efficacy basically means looking at survival, pre-defined adverse events, and the function of the pump. Dwelling on negative aspects that were not the focus of the original study, especially in face of limited journal pages and strict editing, is not done. We must hope that these negative details faced each day by the investigators will be dealt with individually in future reports. Further, much of the design of the study is dictated by the FDA's desire to document adverse events that can be definitively related to the device, hence the subjective division of complications into categories that are easy targets for criticism. Can we always definitively implicate the device or the patient and the disease in bleeding, embolism, infection, end-organ dysfunction, or even death? Obviously we cannot. The data are often subjective and the reports are less than perfect.

We must base our decisions to support or oppose increasing clinical use of artificial hearts on imperfect data. In the end, it seems reasonable to interpret this and similar reports from the perspective of the "big picture." The device pumped physiologic flows, allowed $67 \%$ of a selected group of patients to survive to transplantation with an embolic incidence of $11 \%$, and led to out-of-hospital life for $41 \%$. Some provocative and very important questions of coagulation/bleeding, infection, inflammation, and device durability were raised. These questions must be addressed, but ideally not by studies that are company sponsored. In the meantime, further use of the HeartMate vented electric VAD is indicated.

\section{References}

1. Frazier OH, Rose EA, Oz MC, Dembitsky W, McCarthy P, Radovancevic $B$, et al. Multicenter clinical evaluation of the HeartMate vented electric left ventricular assist system in patients awaiting heart transplantation. J Thorac Cardiovasc Surg. 2001;122:1186-95.

2. Rose EA, Moskowitz AJ, Packer M, Sollano JA, Williams DL, Tierney AR, et al. The REMATCH trial: rationale, design, and end points. Ann Thorac Surg. 1999;67:723-30.

3. Kormos RL, Ramasamy N, Sit S, Cleeland AD, Jassawalla JS, Portner PM. Bridge-to-transplant (BTT) experience with the Novacor left ventricular assist system (LVAS): Results of a multicenter US study. J Heart Lung Transplant. 1999;18:63.

4. Copeland JG 3rd, Arabia FA, Banchy ME, Sethi GK, Foy B, Long J, et al The CardioWest total artificial heart bridge to transplantation: 1993-1996 national trial. Ann Thorac Surg. 1998;66:1662-9.

5. Spanier T, Oz M, Levin H, Weinberg A, Stamatis K, Stern D, et al. Activation of coagulation and fibrinolytic pathways in patients with left ventricular assist devices. J Thorac Cardiovasc Surg. 1996;112: 1090-7.

6. The Novacor IDE. http://www.fda.gov/cdrh/pma/pmasep98.html. Select: Application \#P980012-9/29/98.

7. Minami K, El-Banayosy A, Sezai A, Arusoglu L, Sarnowsky P, Fey $\mathrm{O}$, et al Morbidity and outcome after mechanical ventricular support using Thoratec, Novacor, and HeartMate for bridging to heart transplantation. Artif Organs. 2000;24:421-6.

8. McCarthy PM, Smedira NO, Vargo RL, Goormastic M, Hobbs RE, Starling RC, et al. One hundred patients with the HeartMate ventricular assist device: evolving concepts and technology. J Thorac Cardiovasc Surg. 1998;115:904-12. 